\title{
Glycogen Synthase Kinase-3 $\beta$ (GSK-3 $\beta$ ) is a Critical Factor in Intracellular Signaling Pathways in Acute Lymphoblastic Leukemia
}

\author{
Cristian Fabian Layton Tovar* \\ Autonomous University of Mexico State, Mexico \\ *Corresponding author: Cristian Fabian Layton Tovar, General Agustín Olachea 1401, México
}

Submission: 梅 November 11, 2017; Published: 眥 December 19, 2017

\begin{abstract}
Acute lymphoblastic leukemia (ALL) is a common hematologic malignancy in children, teenagers, and young adults. The ALL development is a complex process, it includes the aberrant gene expression and the presence of chromosomal translocations. Chromosomal rearrangement have incidence in the cell maturation, proliferation and survival process. The treatment of ALL has shown high rates of effectiveness; however, the expression of biomarkers associated with the activation of cell signaling pathways is crucial to establish the prognosis of the disease. Glycogen synthase kinase$3 \beta$ (GSK-3 $\beta$ ) is a member of the serine/tyrosine kinase family with an important role in intracellular pathways signal; the GSK-3 $\beta$ transposition has been associated with changes in the transcriptional activity of nuclear factor kappa B (NF-kB) in blast cells. Nonetheless, others intracellular signaling pathways associated with the GSK-3 $\beta$ activity should participate in the ALL development.
\end{abstract}

Keywords: Acute lymphoblastic leukemia; GSK-3ß; Prognostic

\section{Introduction}

ALL development is associated with deregulation in different points of the molecular signaling pathways associated with the hematopoiesis process. The main determinants of the LLA development of respect to cytogenetic pattern has been established, in this syndrome: $t(1,19), t(4,11), t(12,21)$ and $t(9,22)$ have been described. Moreover, the translocations induced an aberrant expression of the enzymes that regulate the intracellular signaling process [1].

The biomarkers associated with prognosis and treatment response in ALL in the last decade has been extensive. The use of kinase inhibitors of BCR/ABL gene as a molecular target in patients positive to the Philadelphia chromosome ( $\mathrm{Ph}+\mathrm{ALL})$ is available, this occurs with a prevalence of $5 \%$ in children and $30 \%$ in adults. $[2,3]$ or ErbB, a promoter of survival signaling and cell proliferation expressed in B-cell lymphoblast [4,5].

ALL development has been associated with JAK-STAT aberrant expression. JAK2 and JAK1 mutations affect the regulation of intracellular processes of proliferation and survival [6,7]. JAK mutations and CRLF2 over expression in patients with refractory leukemia have been identified and treatment with $J A K$ inhibitors has shown favorable results. Alterations in PI3K-AKT and RasMAPK signaling path way sin hematological malignancies have been evaluated in different populations, hence its role in targeted therapies [8]. The inhibition of rapamycin in mammalian cells (mTOR) involved in the control of the transcription complex has been shown effective in the treatment of pediatric ALL [8-10].

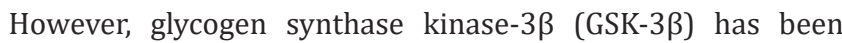
identified as an important regulator of NF-kB transcriptional activity, these effects should regulate the apoptosis in leukemic cells. In this review, there are molecular mechanisms associates with the critical role of GSK-3 $\beta$ in intracellular signaling pathways in ALL.

GSK-3B Regulates NF-Kb Activity in Mononuclear Cells of Bone Marrow

Glycogen synthase kinase (GSK-3) is a serine and tyrosine kinases family identified in skeletal muscle, GSK-3 participates in the culmination of glycogen synthesis. Over expression of GSK-3 has been demonstrated in metabolic disorders and different alterations of the differentiation and proliferation of hematopoietic stem cells [4]. There are two iso forms of GSK-3: the first of $51 \mathrm{kDa}$ (GSK- 3 $\alpha$ ) and the second of $47 \mathrm{kDa}$ (GSK-3 $\beta$ ), Figure 1.

Recently GSK-3 $\beta$ has been associated with the regulation of NF-kB activity. The inhibition of GSK-3 $\beta$ decreases the activation pathway of NF-KB; this inhibition generates gene suppression and stimulates apoptosis in vitro. The importance of targeted molecular 
therapies for each genetic abnormalities related with the process of multiplication of leukemic cells and have emerged in childhood ALL. The isolation of bone marrow aspirates for mononuclear cells has been used to detect GSK-3 $\beta$ by immune fluorescence in primary cell cultures. The inhibition of GSK-3 $\beta$ in vitro showed changes in the transcriptional activity of NF-kB and the induction of apoptosis of leukemic cells. The GSK-3 $\beta$ level was significantly accumulated in the nuclei of all leukemic cells and the cell death induced by the inhibition of GSK-3 $\beta$ was mediated by a down regulation of the transcriptional activity of NF-kB. GSK-3 $\beta$ inhibitors significantly decreased NF-kB expression, it suggesting that it is a new interesting target on ALL treatment [11].

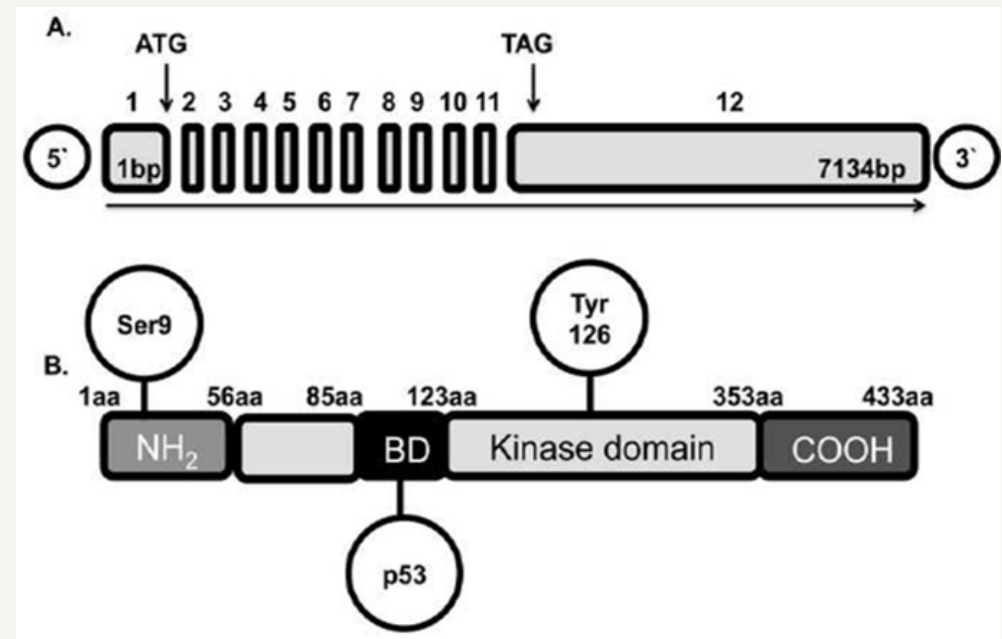

Figure 1: GSK-3 $\beta$ structure. A) The gene is compound of 12 exons. B) The protein has N-terminal and C- terminal extremes, a kinase domain and a BD region associated with a binding site to $\mathrm{p} 53$.

WNT Pathway is a Crucial Regulator of Proliferation and Survival Cell

GSK-3 $\beta$ regulates various signaling pathways for the expression of growth factor and affects a wide range of physiological processes, is an important component in the process of insulin signaling and $W n t$. The relationship between activation pathways and dependent signaling GSK-3 $\beta$ concentrations determined the signaling pathway of insulin exerts a specific action on the glycogen synthase activity. Furthermore, Wnt increases cytosolic expression of beta catenin subunit; the formation of a GSK- $3 \beta$ - axin complex may increase the expression of Wnt through different mechanisms and this activation is very important consider the role of Wht in carcinogenesis and mechanisms of signal transduction [12].

\section{Ribosomal Protein S6 Kinase 1 (S6K1) Regulation is Related with GSK-3 $\beta$ Activity}

In cellular models, GSK-3ßpromoted the activation of p70 subunit of ribosomal protein S6 kinase 1 (S6K1) by phosphorylation. S6K1 plays an important role in cellular processes mediated by insulin sensitivity; these including cell proliferation and proteins synthesis. Therefore, dysregulation of S6K1 contributes to the progression of different metabolic diseases. S6K1 has an important biological and clinical role; moreover is necessary to know the activation processes mediated by GSK- $3 \beta$ activity in the regulation, thus, it is an important regulator of cell proliferation and growth. The results establish the need to develop inhibitors of GSK-3 $\beta$ to treat diseases as diabetes, cancer and other age-related that are linked to inadequate regulation of S6K1 [13].
GSK-3 $\beta$ has been included to determine the pharmacological perspective, therefore intermediate in a number of signaling pathways including insulin/PI3 kinase and Wnt pathway $[13,14]$. GSK-3 $\beta$ Inhibition with lithium or by phosphorylation, activate signaling pathways generally has an apoptotic effect; GSK-3 $\beta$ targets are transcription factors ( $\beta$ - catenin, C -Jun, HSF -1, CREB) and cytoskeletal elements (Tau, MAP1B), determinants involved in metabolic processes. Intracellular GSK- $3 \beta$ should be inhibited by at least five different mechanisms, which are critical for the development of new inhibitors $[15,16]$.

\section{Perspectives}

Thus, in acutelymphoproliferative disorders present in pediatric population, there are not described the molecular biomarkers of disease progression. The use of prognostic factors including biochemical-metabolic, clinical and molecular biomarkers should establish the risk of relapse; approximately half of patients who relapse have a favorable clinical prognosis and excellent response to initial treatment $[17,18]$.

GSK-3 $\beta$ is involved in multiple molecular signaling pathways as PI3K/PTEN/Akt/mTOR and Ras / Raf / MEK / ERK. Moreover, should be considered as a prognostic marker of disease but as a potential target in the development of new treatments that seek to reduce the resistance to current chemotherapy, considering that their aberrant expression has been identified in patients with different types of cancer treatment instituted [19,20].

Inhibitors of GSK- $3 \beta$ have become one of the most powerful tools in the treatment of pathophysiological processes in which the enzyme is an activator of other signaling pathways. The most 
common inhibitors are small synthetic molecules that compete for ATP binding site [15-17]. Recently, Wang has reported that tetramethylpyrazine inhibits the proliferation of ALL cell lines decreasing in GSK-3 $\beta$ signaling, an interesting pharmacological perspective to development [20]. Layton et.al, report that NFKB relative expression levels, in comparison to the GSK-3 $\beta$ immune his to chemistry results of the bone marrow samples, showed a significant difference between positive and negative cases, these results suggest that GSK-3 $\beta$ may be a prognostic biomarker in childhood ALL [21].

\section{References}

1. Winter SS (2011) Pediatric acute leukemia therapies informed by molecular analysis of high-risk disease. Hematology Am Soc Hematol Educ Program 2011: 366-373.

2. Ross ME, Mahfouz R, Onciu M, Liu HC, Zhou X, et al. (2004) Gene expression profiling of pediatric acute myelogenous leukemia. Blood 104(12): 3679-3687.

3. Coustan-Smith E, Sancho J, Behm FG, Hancock ML, Razzouk BI, et al. (2002) Prognostic importance of measuring early clearance of leukemic cells by flow cytometry in childhood acute lymphoblastic leukemia. Blood 2002 100(1): 52-58.

4. Borowitz MJ, Devidas M, Hunger SP, Bowman WP, Carroll AJ, et al. (2008) Clinical significance of minimal residual disease in childhood acute lymphoblastic leukemia and its relationship to other prognostic factors: aChildren's Oncology Group study. Blood 111(12): 5477-5485.

5. Mullighan CG, Phillips LA, Su X, Ma J, Miller CB, et al. (2008) Genomic analysis of the clonal origins of relapsed acute lymphoblastic leukemia. Science 322(5906): 1377-1380.

6. Cario G, Zimmermann M, Romey R, Gesk S, Vater I, et al. (2010) Presence of the P2RY8-CRLF2 rearrangement is associated with a poor prognosis in non-high-risk precursor B-cell acute lymphoblastic leukemia in children treated according to the ALL-BFM 2000 protocol. Blood 115(26): 5393-5397.

7. Bercovich D, Ganmore I, Scott LM, Wainreb G, Birger Y, et al. (2008) Mutations of JAK2 in acute lymphoblastic leukaemias associated with Down's syndrome. Lancet 372(9648): 1484-1492.

8. Lee-Sherick AB, Linger RM, Gore L, Keating AK, Graham DK, et al. (2010) Targeting paediatric acute lymphoblastic leukaemia: novel therapies currently in development. Br J Haematol 151(4): 295-311.

9. Hidalgo M, Rowinsky EK (2000) The rapamycin-sensitive signal transduction pathway as a target for cancer therapy. Oncogene 19(56): 6680-6686

10. Avellino R, Romano S, Parasole R, Bisogni R, Lamberti A, et al. (2005) Rapamycin stimulates apoptosis of childhood acute lymphoblastic leukemia cells. Blood 106(4): 1400-1406.

11. Hu Y, Gu X, Li R, Luo Q, Xu Y, et al. (2010) Glycogen synthase kinase3beta inhibition induces nuclear factor-kappa B-mediated apoptosis in pediatric acute lymphocyte leukemia cells. Journal of experimental \& clinical cancer research 29: 154.

12. Ding VW, Chen RH, Cormick MF (2010) Differential regulation of glycogen synthase kinase 3beta by insulin and Wnt signaling. J Biol Chem 275(42): 32475-32481.

13. Shin S, Wolgamott L, Yu Y, Blenis J, Yoon SO, et al. (2011) Glycogen synthase kinase (GSK)-3 promotes p70 ribosomal protein S6 kinase (p70S6K) activity and cell proliferation. Proc Natl Acad Sci USA 108(47): E1204-E1213.

14. Dann SG, Selvaraj A, Thomas G (2007) mTOR Complex1-S6K1 signaling: at the crossroads of obesity, diabetes and cancer. Trends Mol Med 13(6): 252-259.

15. Gould TD, Quiroz JA, Singh J, Zarate CA, Manji HK, et al. (2004) Emerging experimental therapeutics for bipolar disorder: insights from the molecular and cellular actions of current mood stabilizers. Mol Psychiatry 9(8): 734-755.

16. Jope RS, Johnson GV (2004) The glamour and gloom of glycogen synthase kinase- 3. Trends Biochem Sci 29(2): 95-102.

17. Carnevalli LS, Masuda K, Frigerio F, Bacquer LO, Um SH, et al. (2010) S6K1 plays a critical role in early adipocyte differentiation. Dev Cell 18(5): 763-774.

18. Lacayo NJ, Meshinchi S, Kinnunen P, Yu R, Wang Y, et al. (2004) Gene expression profiles at diagnosis in de novo childhood AML patients identify FLT3 mutations with good clinical outcomes. Blood 104(9): 2646-2654.

19. Duvel K, Yecies JL, Menon S, Raman P, Lipovsky AI, et al. (2010) Activation of a metabolic gene regulatory network downstream of mTOR complex 1. Mol Cell 39(2): 171-183.

20. Wang XJ, Xu YH, Yang GC, Chen HX, Zhang P, et al. (2015) Tetramethylpyrazine inhibits the proliferation of acute lymphocytic leukemia cell lines via decrease in GSK-3ß. Oncol Rep 33(5): 2368-2374.

21. Layton CF, Mendieta H, Camarillo MC, Fabila YF, Tejocote I, et al. (2016) Glycogen Synthase Kinase-3 $\beta$ (GSK-3 $\beta$ ) and Nuclear Factor Kappa-B (NFKB) in Childhood Acute Lymphoblastic Leukemia. Adv Clin Exp Med 25(6): 1139-1147. 